\title{
Performance of Iterative Soft Decision Feedback Equalizers for Single-Carrier Transmission
}

\author{
Taehyun Jeon*, Seokhyun Yoon** and Kyungho Kim ${ }^{\dagger}$
}

\begin{abstract}
In this paper, we consider iterative soft-decision feedback equalizers (sDFE), a.k.a. turbo equalizers for single-carrier transmission. Turbo equalizer takes log-likelihood ratio (LLR) feedback from channel decoder and convert the LLR into symbol estimates and variances to be used for the LLR update at the sDFE. Specifically, we consider both time domain and frequency-domain sDFE and compare their performances. The results shows that frequency-domain sDFE performs better than time-domain one and also that considerable gain can be obtained especially when the channel has deep nulls.
\end{abstract}

Keywords: Turbo equalization, Soft decision feedback equalizer, Frequency domain equalizer

\section{Introduction}

Single-carrier (SC) transmission has been adopted in many wireless standards, such as LTE uplink, IEEE 802.11ad and its extension 802.11ay. One problem of SC transmission is that its performance can be degraded when channel has deep nulls. The problem in the channel with deep null is that it requires relatively long equalizer tap coefficients. Frequency domain equalizer $[1,2]$ is a good candidate since it works well even when the channel has deep nulls, even though it still suffers from the noise enhancement. In this sense, decision feedback equalizers (DFE) [3-5] can be considered to combat the channels with deep null since it not only can mitigate the noise enhancement, but also can reduce the equalizer length by utilizing the feedback filters which is effectively an IIR filter. One of the problems in DFE, however, is the error propagation, especially when we use the immediate decision made by symbol de-mapper.

Recently, iterative detection and decoding schemes [611] that utilize the soft-decisions provided by iterative channel decoders have been considered with technology development in low cost computing hardware. Especially, in [6], maximum likelihood sequence estimator (MLSE) is used for the soft-in/soft-out detection, while those in [7-11] utilize soft-decision feedback and interference cancellation. In complexity point of view, MLSE has a complexity exponentially increasing with the channel length, and the scheme in [6] is not suitable for practical application even though it generally outperforms all other schemes. In this sense, the soft-decision feedback cancellation schemes in

$\uparrow$ Corresponding Author: Dept. of Electronic and Electrical Eng., Dankook University, Korea. (dkuhealth@dankook.ac.kr)

* Dept. of Electrical and Information Engineering, Seoul National University of Science and Technology, Korea. (thjeon@seoultech.ac.kr)

** Dept. of Electronic and Electrical Eng., Dankook University, Korea. (syoon@dku.edu)

Received: August 1, 2016; Accepted: January 17, 2017
[7-11] look more feasible for practical application at a reasonable computational cost.

Based on this observations, we, in this paper, consider to use turbo equalization techniques using the decision feedback equalizer in time-domain [2,3] and in frequency domain $[10,11]$ by replacing the immediate hard-decision with the soft-decisions fed-back from channel decoder. Using log-likelihood ratio (LLR) feedback from channel decoder, we extract the soft decision as a posteriori mean and the reliability information as a posteriori variance, which are then used to cancel the residual interference. The main focus in this paper is a comparison of these iterative equalization with time-domain and frequency domain DFE.

The paper is organized as follows: In the next section, we briefly review the system model and a general structure of turbo equalizer. In section III, we provide a brief description on soft-decision feedback equalizer, both in time-domain and frequency domain. In section IV, we compare the performance of the two turbo equalizers for a fixed channel with deep nulls. Finally, the concluding remarks are given in section $\mathrm{V}$.

\section{System Description}

In this section, we provide a brief description of the system we consider, including frame structure, signal model, and a generic structure of turbo equalizer.

\subsection{Frame structure and frequency domain equalizer}

A discrete-time multipath inter-symbol interference (ISI) channel is modeled as

$$
y_{k}=\sum_{d=0}^{L-1} h_{d} x_{k-d}+n_{k}
$$




\begin{tabular}{c|c|c|c|c|c|}
\multicolumn{1}{c}{ Gl } & Data & \multicolumn{1}{c}{ Gl } & Data & \multicolumn{1}{c}{$\mathrm{Gl}$} \\
\hline$\ldots$. & $P$ & $N$-PSymbols & $P$ & $N-P$ Symbols & $P$ \\
\hline
\end{tabular}

Fig. 1. The data portion of the frame structure under consideration; a fixed postfix of length $P$ samples is periodically inserted at the end of $N-P$ data symbols and also added at the beginning of a frame such that it is positioned on both sides

where $h_{d}$ is the discrete channel impulse response of length $L, y_{k}$ the received signal, $x_{k}$ the transmitted data drawn from an alphabet set, $\Xi$, of size $2^{m}$, and $n_{k}$ the noise sequence. The noise sequence is assumed to have mean 0 and the auto-covariance, $E\left[n_{k}{ }^{*} n_{k-d}\right]=\sigma^{2} \delta_{d}$ and the data to have mean 0 and the auto-covariance, $E\left[x_{k}^{*} x_{k-d}\right]=\delta_{d}$.

Fig. 1 shows frame structure of a general single carrier transmission, where data stream is divided into blocks of length $N-P$ symbols and fixed postfixes of length $P$ are inserted between each data block (For the first block, it is also added at the beginning of the block). Utilizing this structure, iterative decision feedback equalization can be applied in block-by-block fashion. Let us define a transmission block as one data block (of $N-P$ symbols) followed by the fixed postfix (of length $P$ ), respectively. Due to the periodicity of the postfixes, one can use blockwise signal model, i.e.,

$$
\mathbf{y}=\tilde{\mathbf{H}} \mathbf{x}+\mathbf{n}
$$

where $\mathbf{y}$ is $N \times 1$ received signal vector, $\mathbf{x} N \times 1$ vector of a transmission block, and $\mathbf{n}$ is $N \times 1$ noise vector with mean $\mathbf{0}$ and covariance $E\left[\mathbf{n n}^{H}\right]=\sigma^{2} \mathbf{I}$. The channel matrix is $N \times N$ circulant matrix, of which the $k$ th column is given by the circular shift by $(k-1)$ of the vector

$$
\mathbf{h}=\left[h_{0}, h_{1}, \ldots, h_{L-1}, 0, \ldots, 0\right]^{T}
$$

of size $N \times 1$. As an example for $N=7$ and $L=3 \leq P$, it is given by

$$
\tilde{\mathbf{H}}=\left[\begin{array}{ccccccc}
h_{0} & 0 & 0 & 0 & 0 & h_{2} & h_{1} \\
h_{1} & h_{0} & 0 & 0 & 0 & 0 & h_{2} \\
h_{2} & h_{1} & h_{0} & 0 & 0 & 0 & 0 \\
0 & h_{2} & h_{1} & h_{0} & 0 & 0 & 0 \\
0 & 0 & h_{2} & h_{1} & h_{0} & 0 & 0 \\
0 & 0 & 0 & h_{2} & h_{1} & h_{0} & 0 \\
0 & 0 & 0 & 0 & h_{2} & h_{1} & h_{0}
\end{array}\right]
$$

Linear frequency domain equalizer: Since the channel matrix is circulant, it can be decomposed into the form of $\tilde{\mathbf{H}}=\mathbf{E} \mathbf{G E}^{H}$ where $\mathbf{E}$ is DFT matrix and $\mathbf{G}$ is a diagonal matrix such that $\operatorname{diag}(\mathbf{G})=\mathbf{E h}$ where $\mathbf{E h}$ represents the

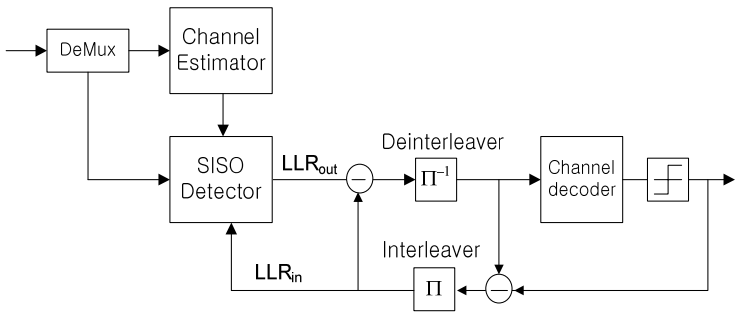

Fig. 2. Generic structure of iterative detection and decoding for single-carrier transmission through ISI channel

channel frequency response. That means the channel can be perfectly equalized in frequency domain if there is no nulls in $\operatorname{diag}(\mathbf{G})$. Unfortunately, however, if the values in $\operatorname{diag}(\mathbf{G})$ is very small compared to the noise power, noise is enhanced by the equalization itself.

There is an MMSE version of frequency equalization which minimizes the noise enhancement. That is, in frequency domain, we use for each sub-carrier a one-tap equalizer given by $g_{j}^{*} /\left(\left|g_{j}\right|^{2}+\sigma^{2}\right)$ for the $j$ th sub-carrier. Although the overall noise + interference can be minimized and linear MMSE equalizer, either in time domain or in frequency domain, works well in most cases in WLAN environment, there still exists residual ISI in time domain signal and it might have poor performance, especially when the channel has deep nulls. Such condition often occur in wireless environment, and, sometimes, we may need more powerful receiver algorithm, e.g., turbo equalizers, to minimize the outage probability.

\subsection{Turbo equalizer: A generic structure}

Fig. 2 shows a generic structure of turbo equalizer, where one of the key components is the soft-in/soft-out (SISO) detector. It takes two inputs, one from the output of the feed-forward filter and the other from the channel decoder, i.e., LLRs for each coded bit. The bit-LLR is initialized to zero at the first iteration. A typical SISO detector is the MLSE as in [6], where the forward and backward algorithm is used for soft-in/soft-out detection. Although it is the best choice in error performance, its complexity is proportional to $O\left(2^{m L}\right)$, which is prohibitive even with a moderate channel length.

Another choice for the SISO detector is the soft decision feedback equalizer(sDFE), which uses soft decisions fedback from the channel decoder to cancel out the intersymbol interferences caused by multi-path propagation. In this paper, we focus on such a turbo equalizer, specifically either with time domain sDFE or with frequency domain sDFE.

\section{Soft Decision Feedback Equalizers}

In this section, we consider two types of sDFE, one 


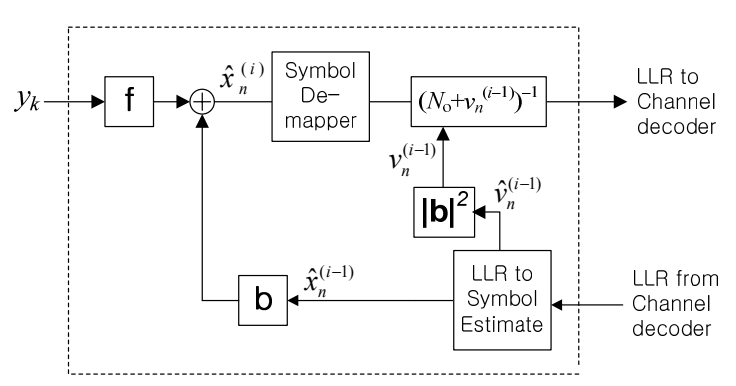

Fig. 3. Time domain soft-decision feedback equalizer

operating in time-domain and the other in frequency domain. Although there are many other options with minor variations, we specifically considers DFE in [4] for timedomain sDFE and [11] for frequency-domain sDFE as they are better suited to our framework.

\subsection{Time domain soft-decision feedback equalizer}

Fig. 3 shows time domain soft-decision feedback equalizer (sDFE) with LLR feedback from channel decoder. The sDFE in Fig. 3 consists of a feed-forward filter (denoted as a vector $f$ ) and a feedback filter (denoted as a vector $b$ ). The latter takes the output of 'LLR to symbol estimates' module, which converts the LLR from channel decoder into a pair of symbol estimate, $\hat{x}_{n}^{(i)}$, and its variance, $\hat{v}_{n}^{(i)}$, while the former takes the received signal, $y_{k}$, as its input. The output of the feedforward filter is subtracted by the output of the feedback filter before being passed to the symbol de-mapper. Specifically, the reestimated data symbol at the $i$ th iteration can be expressed as follows

$$
\hat{x}_{k}^{(i)}=\sum_{d=0}^{N_{f}-1} f_{d} y_{k-d}-\sum_{d=0}^{N_{b}-1} b_{d} \hat{x}_{k-d}^{(i-1)}
$$

where $N_{f}$ and $N_{b}$ are the length of feed-forward filter and the feedback filter, respectively.

The coefficients of the feed-forward filter, $f_{d}$, and that of the feedback filter, $b_{d}$, can be obtained in the same way as in [3-5] as follows: Define the channel matrix $\mathbf{H}$ of size $N_{f} \times\left(N_{f}+L-1\right)$

$$
\mathbf{H}^{T}=\left[\begin{array}{cccccccc}
h_{0} & h_{1} & \cdots & h_{L-1} & 0 & \cdots & \cdots & 0 \\
0 & h_{0} & h_{1} & \cdots & h_{L-1} & 0 & \cdots & 0 \\
\vdots & & & & & & & \vdots \\
0 & \cdots & \cdots & 0 & h_{0} & h_{1} & \cdots & h_{L-1}
\end{array}\right]
$$

This is different from (3), i.e., (3) is for the block signal model with a fixed postfix, while the above is for linear model. For a given channel matrix $\mathbf{H}$, define its covariance matrix $\mathbf{K}$ as

$$
\mathbf{K}=\sigma^{2} \mathbf{I}+\mathbf{H}^{H} \mathbf{H}
$$

which can be factorized by Cholesky decomposition into

$$
\mathbf{K}=\mathbf{L D L}^{H}
$$

where $\mathbf{L}$ is a lower triangular matrix of size $\left(N_{f}+L-1\right) \times$ $\left(N_{f}+L-1\right)$ and $\mathbf{D}$ is a diagonal matrix of the same size. Let $d_{n}$ be the $n$th diagonal element of $\mathbf{D}$ and $\mathbf{e}_{n}$ be a vector with its $n$th element being 1 and all others being 0 . Then, defining $\mathbf{u}$ and $\mathbf{w}$ as

$$
\begin{gathered}
\mathbf{u}=d_{N_{f}-1}^{-1} \mathbf{e}_{N_{f}}^{T} \mathbf{L}^{-1} \mathbf{H}^{H} \\
\mathbf{w}=\mathbf{L} \mathbf{e}_{N_{f}},
\end{gathered}
$$

the impulse responses of the feed-forward filter, $f_{k}$, and the feedback filter, $b_{k}$, are given respectively by [4]

$$
\begin{gathered}
f_{k}=u_{-k} \\
b_{k}=\delta_{k}-w_{k}
\end{gathered}
$$

Since $\mathbf{L}$ is lower triangular matrix and $\mathbf{b}$ is $N_{f}$ th column of $\mathbf{L}, \mathbf{b}$ has only $L$ non-zero elements resulting in its effective length being $N_{b}=L$, i.e., the length of feedback filter is equal to or less than $L$, the channel length.

In the original decision feedback equalizer in [4-5], the decision is made based on the output of symbol demapper. Although the operation is simple in this case, error propagation could be a problem since the immediate decision from the symbol de-mapper might contain frequent errors. Therefore, we resort the LLRs from channel decoder, which is more reliable for ISI cancellation, minimizing the error propagation.

To utilize the feedback from the channel decoder, which outputs LLR for each coded bit, we need to convert the LLR into symbol estimates and its variance to take the reliability information into account. The procedure to generate the LLR to be passed to the channel decoder is also depicted in Fig. 2, where (1) the DFE takes LLR from channel decoder, (2) converts it into symbol estimate $\hat{x}_{n}^{(i)}$ and the estimation error variance $\hat{v}_{n}^{(i)}$, (3) generates an updated symbol estimate $\hat{x}_{n}^{(i)}$, (4) convert it into LLRs (using symbol de-mapper) and, (5) finally, fed them back to channel decoder. Since most SC transmission schemes utilize fixed prefix for each block, one can use the fixed prefix as initial decisions with variance 0 and the equalization can be performed per-block basis.

The conversion of LLR (from channel decoder) to symbol estimates and its estimation error variance is as follows: Consider $2^{m}$-ary modulation, where a modulation symbol can be represented as a function of data bits contained in a symbol. Letting $b_{n k}$ be the $k$ th bit contained in $x_{n}$, we can represents the modulation symbol as $x_{n}\left(b_{n 1}, b_{n 2}, \ldots, b_{n 2^{m}}\right)$ and, for given LLR $L_{n k}$ of $b_{n k}$, we have

$$
p\left(b_{n k}= \pm 1\right)=\frac{e^{ \pm L_{n k} / 2}}{e^{+L_{n k} / 2}+e^{-L_{n k} / 2}}
$$


Assuming $b_{n k}$ 's are independent of each other, the symbol likelihood is given then by

$$
p\left(x_{n}\right)=\prod_{k=1}^{2^{m}} p\left(b_{n k}\right)
$$

and the symbol estimates and its variance are obtained by taking expectation over the symbol likelihoods, i.e.,

$$
\begin{gathered}
\hat{x}_{n}=E\left[x_{n}\right]=\sum_{x_{n} \in \Xi} x_{n} p\left(x_{n}\right) \\
\hat{v}_{n}=\operatorname{Var}\left[x_{n}\right]=\sum_{x_{n} \in \Xi}\left|x_{n}\right|^{2} p\left(x_{n}\right)-\left|\hat{x}_{n}\right|^{2}
\end{gathered}
$$

where we omitted the superscript representing the iteration number for notational simplicity. Note that, using the independence of $b_{n k}$ 's, we have for QPSK

$$
\begin{aligned}
& \hat{x}_{n}=\frac{1}{\sqrt{2}} \tanh \left(\frac{L_{n 1}}{2}\right)+\frac{j}{\sqrt{2}} \tanh \left(\frac{L_{n 2}}{2}\right) \\
& \hat{v}_{n}=1-\frac{1}{2} \tanh ^{2}\left(\frac{L_{n 1}}{2}\right)-\frac{1}{2} \tanh ^{2}\left(\frac{L_{n 2}}{2}\right)
\end{aligned}
$$

where we assumed odd-indexed bits are mapped to inphase component while even-indexed to quadrature-phase component. The estimation error variance, $\hat{v}_{n}$, is added to the background noise power, $N_{0}$, and used to weigh the symbol de-mapper output, i.e., by $\left(N_{0}+v_{k}\right)^{-1}$ with

$$
N_{0}=\sigma^{2} \sum_{d=0}^{N_{f}-1}\left|f_{d}\right|^{2} \text { and } v_{k}=\hat{v}_{k} \sum_{d=0}^{N_{b}-1}\left|b_{d}\right|^{2}
$$

On the other hand, conversion of symbol estimates to LLR is based on the posterior probability given by

$$
\hat{p}\left(x_{n}^{(i)}=x\right) \propto \exp \left(-\frac{\left|\tilde{x}_{n}^{(i)}-x\right|^{2}}{2 \hat{\sigma}_{n}^{2}}\right)
$$

where $\hat{\sigma}_{n}^{2}$ is the effective noise power given by $N_{0}+v_{k}$. And the posterior LLR can be computed by

$$
\begin{gathered}
L\left(b_{n k}\right)=\log \frac{\sum_{\text {all } x: b_{n k}=0} \hat{p}\left(x_{n}^{(i)}=x\right)}{\sum_{\text {all } x: b_{n k}=1} \hat{p}\left(x_{n}^{(i)}=x\right)} \\
\approx \frac{1}{2 \hat{\sigma}_{n}^{2}}\left(\min _{\text {all } x: b_{n k}=0}\left|\tilde{x}_{n}^{(i)}-x\right|^{2}-\min _{\text {all } x: b_{n k}=1}\left|\tilde{x}_{n}^{(i)}-x\right|^{2}\right)
\end{gathered}
$$

\subsection{Frequency domain soft-decision feedback equalizer}

Fig. 4 shows the frequency domain sDFE under consideration. Similar to the time-domain sDFE, it also has a feed-forward filter denoted as $F$, a feedback filter denoted as $B$, a symbol de-mapper converting the symbol estimates,

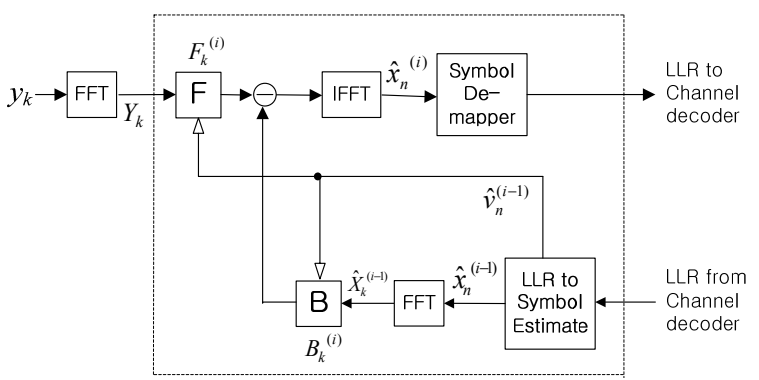

Fig. 4. Frequency domain soft-decision feedback equalizer

$\hat{x}_{n}^{(i)}$ into LLR and the 'LLR to symbol estimates' module. The difference from the time-domain SDFE is that the feedforward filtering and feedback filtering are performed in frequency domain. Here, we first perform $N$-point DFT to the received signal, $y_{k}$, where $k=0,1,2, \ldots, N-1$, i.e., for a block of length $N$ including the postfix. Then we can write the received signal in frequency domain as

$$
Y_{k}=H_{k} X_{k}+N_{k}, \quad k=0,1, \ldots, N-1
$$

where $X_{k}$ is the DFT of one transmission block (of $N$ symbols), $H_{k}$ is the channel frequency response and $N_{k}$ is the noise samples in frequency domain. The estimated signal at the $i^{\text {th }}$ iteration is given by the following expression in frequency domain (per sub-carrier)

$$
\hat{X}_{k}^{(i)}=F_{k}^{(i)} Y_{k}-B_{k}^{(i)} \hat{X}_{k}^{(i-1)}
$$

The frequency domain feed-forward filter coefficients at the $i^{\text {th }}$ iteration (denoted as $F_{k}{ }^{(i)}$ ) and the feedback filter coefficients (denoted as $B_{k}{ }^{(i)}$ ), that maximize the SINR can be obtained in the same way as in [10] as follows.

$$
\begin{gathered}
F_{k}^{(i)}=\frac{H_{k}^{*}}{\sigma_{n}{ }^{2}+\left(1-\left(\rho^{(i-1)}\right)^{2}\right)\left|H_{k}\right|^{2}} \\
B_{k}{ }^{(i)}=\rho^{(i-1)}\left(F_{k}{ }^{(i)} H_{k}-r^{(i)}\right)
\end{gathered}
$$

where

$$
\begin{aligned}
& \left.r^{(i)}=E\left[F_{k}^{(i)} H_{k}\right]=\frac{1}{N} \sum_{k=0}^{N-1} F_{k}^{(i)} H_{k}\right], \\
& \rho^{(i-1)}=E\left[X_{k}^{*} \hat{X}_{k}^{(i-1)}\right]
\end{aligned}
$$

In (24), $\rho^{(i-1)}$ is the correlation coefficient between the original data and the estimated ones, which takes a value from 0 to 1 and typically approaches to 1 , as iteration continue. It should be estimated to properly implement the turbo equalizer and can be simply estimated from the average bit error rate $\hat{P}_{e}^{(i)}$ as

$$
\hat{\rho}^{(i-1)}=1-2 \hat{P}_{e}^{(i-1)}
$$


It can be shown from [10] that the average bit error rate $\hat{P}_{e}^{(i)}$ can be approximated by

$$
\hat{P}_{e}^{(i-1)} \approx Q\left(\sqrt{\frac{\sigma_{s}^{2}}{\hat{v}_{n}^{(i-1)}}}\right)
$$

where, $\sigma_{s}^{2}$ is the signal power, $\hat{v}_{n}{ }^{(i-1)}$ is the estimation error variance defined as in (15) and $Q(\cdot)$ is defined as

$$
Q(x)=\frac{1}{\sqrt{2 \pi}} \int_{x}^{\infty} e^{-\frac{t^{2}}{2}} d t
$$

Note that, when $i=0$, we have $\rho^{(0)}=0,(23)$ becomes

$$
F_{k}^{(i)}=K^{(i)} \frac{H_{k}{ }^{*}}{\sigma_{n}{ }^{2}+\left|H_{k}\right|^{2}} \text { and } B_{k}^{(i)}=0
$$

which is nothing but the frequency domain LMMSE equalizer without feedback. Hence, at the first iteration, the sDFE is effectively the same as MMSE FDE.

\section{Performance Evaluation}

In this section, we evaluate the performance of the two turbo equalizers, one with time-domain SDFE and the other with frequency-domain sDFE, for a channel with deep nulls. As references, we also compare the performance of the linear MMSE receivers both in time and frequency domain. For channel code, we used DVB-S2 LDPC code, which is a capacity approaching code of length 64800, with a code rate $1 / 2$. Note that we set the inner iterations for the decoding of LDPC code to 50 for MMSE TDE/FDE, while for iterative detection and decoding we set it to 25 in order to minimize the computational complexity. Note here that, with this setting, the computational burden for channel decoding at the second turbo iteration is the same as those of linear equalizers without turbo iteration.

We consider a channel with fixed coefficients given by the following discrete time channel impulse response

$$
h_{n}=h_{0} \delta_{n}+h_{1} \delta_{n-5}+h_{2} \delta_{n-10}
$$

We generated many random channel coefficients and selected one with deep nulls, which are $h_{0}=-0.5758+$ $0.1410 j, h_{1}=0.7495-0.0912 j$ and $h_{2}=-0.0979-0.2624 j$, respectively.

Fig. 5 and 6 show the performance comparison for QPSK and 16QAM, respectively. The figures clearly show the performance improvement with the number of turbo iterations denoted as $i 2, \mathrm{i} 3, \ldots$ for sDFE. As mentioned, the performance of linear MMSE receivers, denoted as LTDE and LFDE, can be regarded as the performance of sDFE at the first iteration. In Fig. 5, it can be seen that the SNR improvement by using the turbo equalizers with timedomain and frequency-domain sDFE from that of linear MMSE FDE is approximately 0.5 and $0.9 \mathrm{~dB}$, respectively. In Fig. 6 for 16QAM, the improvement is even larger than that for QPSK, i.e., $1.5 \mathrm{~dB}$ gain with time-domain sDFE and $2.9 \mathrm{~dB}$ with frequency-domain sDFE, respectively.

From Fig. 5 and 6, one also can observe that a considerable gain can be obtained with only 2 or 3 turbo iterations. Although, with 3 iterations, we need 3 times of FFT/iFFT and LDPC decoding of that without turbo iterations, the overall complexity is less than 3 times with the above setting of the number of iterations for the LDPC decoding. As mentioned before we set the inner iterations for the decoding of LDPC code to 50 for MMSE TDE/FDE, while for iterative detection and decoding we set it to 25 .

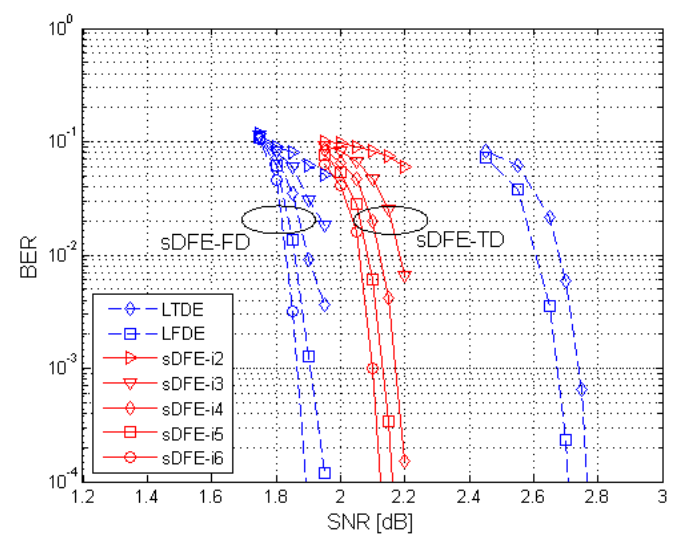

Fig. 5. A comparison of BER performances of (1) time domain LMMSE equalizer (LTDE), (2) frequency domain LMMSE equalizer (LFDE), (3) time domain sDFE and (4) frequency domain sDFE, respectively. The number of turbo iterations for sDFE-TD/FD is 2, 3, 4, 5 and 6; QPSK modulation

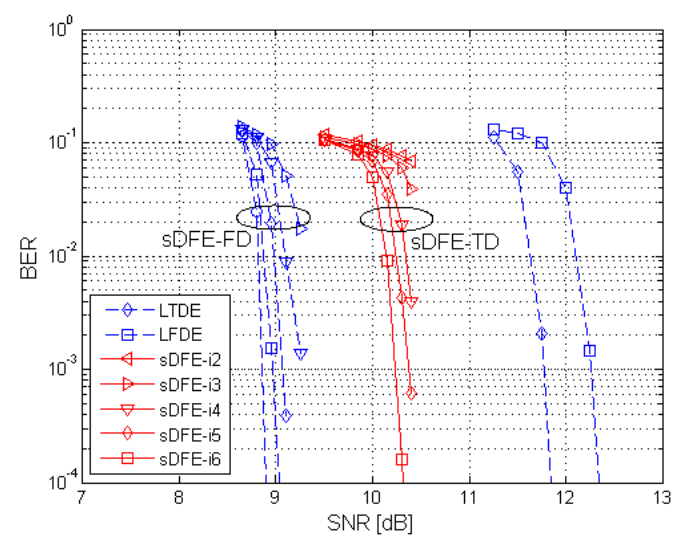

Fig. 6. A comparison of BER performances of (1) time domain LMMSE equalizer (LTDE), (2) frequency domain LMMSE equalizer (LFDE), (3) time domain SDFE and (4) frequency domain SDFE, respectively. The number of turbo iterations for sDFE-TD/FD is 2, 3, 4, 5 and 6; 16QAM modulation 
Hence, for 3 turbo iterations, the complexity increase in LDPC decoding is only 1.5 times. Although the complexity for FFT/iFFT is 3 times (with 3 turbo iterations), it is much smaller than that for LDPC decoding. Note also that, for 2 turbo iteration, the increase in complexity is doubled only for FFT/iFFT and the complexity increase can be negligible.

Another thing to note is that since the occurrence of deep-null is not so often especially in WLAN environment, the overall computational complexity can be minimized by properly managing the number of iterations according to the channel states. This does not mean, however, that the iterative detection and decoding scheme is useless, as they can reduces the outage probability, keeping users being connected even when the channel has deep nulls.

\section{Conclusion}

In this paper, we considered turbo equalizers utilizing soft-decision feedback equalizers (sDFE). We considered both time-domain and frequency-domain sDFE and compared their performance. Simulation results shows that frequency domain sDFE performs better than timedomain one and also that considerable gain can be obtained especially when the channel has deep nulls. Although the computational complexity increases, it is not so big and one can properly manage the number of iterations according to the channel states to compromise the computational complexity and the performance.

\section{Acknowledgements}

This work was supported by Basic Science Research Program through the National Research Foundation of Korea (NRF) funded by the Ministry of Education, Science and Technology (NRF-2012R1A1A2038807)

\section{References}

[1] D. Falconer, S.L. Ariyavisitakul, A. Benyamin-Seeyar, and B. Eidson, "Frequency Domain Equalization for Single-carrier Broadband Wireless Systems," IEEE Commun. Mag., vol. 40, no. 4, pp. 58-66, Apr. 2002.

[2] F. Pancaldi, G. M. Vitetta, R. Kalbasi, N. Al-Dhahir, M. Uysal, and H. Mheidat, "Single-Carrier Frequency Domain Equalization," IEEE Sig. Proc. Mag., pp.3756, Sep. 2008

[3] I. Lee and J. M. Cioffi, "A Fast Computation Algorithm for the Decision Feedback Equalizer," IEEE Trans. Comm., pp. 2742-2749, Nov. 1995.

[4] N. M. W. Al-Dhahir and J. M. Cioffi, "MMSE decision-feedback equalizers: Finite Length Results," IEEE Trans. Inform. Theory, pp. 961-975, July 1995.
[5] A. Duel-Hallen, "A Family of Multiuser DecisionFeedback Detectors for Asynchronous Code-Division Multiple-Access Channels," IEEE Trans. on Commun., vol. 43, no. 2/3/4, pp. 421-434, Feb./March/April 1995

[6] M. Tüchler, R. Koetter, and A. C. Singer, "Turbo Equalization: Principles and New Results," IEEE Trans. Commun., vol. 50, no. 5, pp. 754-767, 2002.

[7] A. M. Chan and G. W. Wornell, "A Class of Blockiterative Equalizers for Intersymbol Interference Channels: Fixed Channel Results," IEEE Trans. Commun., vol. 49, no. 11, pp. 1966-1976, Nov. 2001.

[8] R. R. Lopes and J. R. Barry, "Soft-output Decisionfeedback Equalization with a Priori Information," in IEEE Global Comm. Conference (GLOBECOM '03), vol. 3, pp. 1705-1709, San Francisco, Dec. 2003

[9] R. Dinis, A. Gusmao, and N. Esteves, "On Broadband Block Transmission over Strongly FrequencySelective Fading Channels," in Proc. Wireless 2003, Calgary, Canada, July 2003.

[10] N. Benvenuto and S. Tomasin, "Block Iterative DFE for Single Carrier Modulation," IEE Electron. Lett., vol. 38, pp. 1144-1145, Sept. 2002.

[11] N. Benvenuto and S. Tomasin, "Iterative Design and Detection of a DFE in the Frequency Domain," IEEE T. Comm., vol. 53, no.11, pp.1867-1875, Nov. 2005.

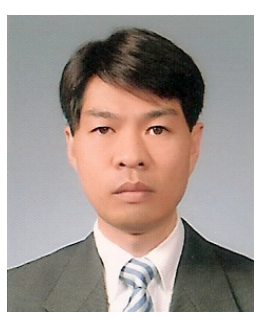

Taehyun Jeon received B.S. degree in Electrical Engineering from Yonsei University, Korea, in 1989, and his M.S. and Ph.D. degrees in Electrical Engineering from University of Minnesota in 1993 and 1997, respectively. His research interests are communication systems and signal processing.

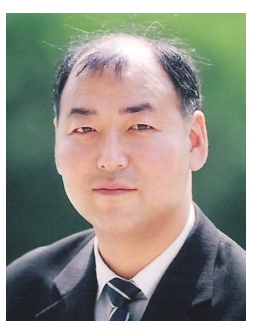

Seokhyun Yoon received his B.S. and M.S. degrees in Electronics Engineering from Sung Kyun Kwan University, Korea, in 1992 and 1996, respectively, and his Ph.D. degree in Electrical and Computer Eng. from the New Jersey Institute of Technology, Newark, in 2003. His research interests are wireless communications and signal processing.

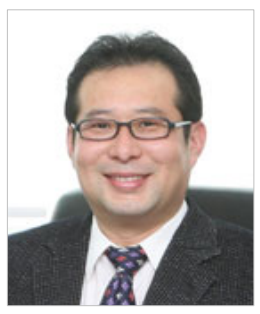

Kyungho Kim received M.S. and Ph. D. degrees in biomedical engineering from Keio University, Japan. He worked at SAIT from 2000 to 2006 . He is currently a professor at Dankook University. His research interests are ubiquitous health, signal processing, sensor system and its applications. 